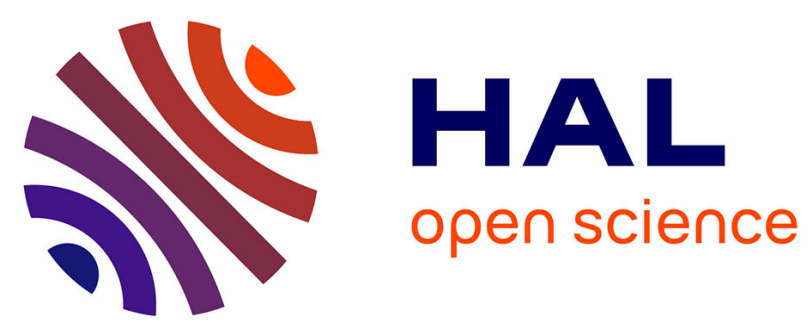

\title{
AlN photonic crystal nanocavities realized by epitaxial conformal growth on nanopatterned silicon substrate
} Delphine Néel, Sylvain Sergent, Meletios Mexis, Diane Sam-Giao, Thierry Guillet, Christelle Brimont, Thierry Bretagnon, Fabrice Semond, B. Gayral, Sylvain David, et al.

\section{To cite this version:}

Delphine Néel, Sylvain Sergent, Meletios Mexis, Diane Sam-Giao, Thierry Guillet, et al.. AlN photonic crystal nanocavities realized by epitaxial conformal growth on nanopatterned silicon substrate. Applied Physics Letters, 2011, 98 (26), pp.261106. 10.1063/1.3605592 . hal-00632917

\section{HAL Id: hal-00632917 https://hal.science/hal-00632917}

Submitted on 25 May 2021

HAL is a multi-disciplinary open access archive for the deposit and dissemination of scientific research documents, whether they are published or not. The documents may come from teaching and research institutions in France or abroad, or from public or private research centers.
L'archive ouverte pluridisciplinaire HAL, est destinée au dépôt et à la diffusion de documents scientifiques de niveau recherche, publiés ou non, émanant des établissements d'enseignement et de recherche français ou étrangers, des laboratoires publics ou privés. 


\section{AIN photonic crystal nanocavities realized by epitaxial conformal growth on nanopatterned silicon substrate}

Cite as: Appl. Phys. Lett. 98, 261106 (2011); https://doi.org/10.1063/1.3605592

Submitted: 01 April 2011 . Accepted: 09 June 2011 . Published Online: 29 June 2011

D. Néel, S. Sergent, M. Mexis, D. Sam-Giao, T. Guillet, C. Brimont, T. Bretagnon, F. Semond, B. Gayral, S. David, X. Checoury, and P. Boucaud

ARTICLES YOU MAY BE INTERESTED IN

High quality factor two dimensional GaN photonic crystal cavity membranes grown on silicon substrate

Applied Physics Letters 100, 071103 (2012); https://doi.org/10.1063/1.3684630

High quality factor AIN nanocavities embedded in a photonic crystal waveguide

Applied Physics Letters 100, 191104 (2012); https://doi.org/10.1063/1.4712590

High-Q AIN photonic crystal nanobeam cavities fabricated by layer transfer

Applied Physics Letters 101, 101106 (2012); https://doi.org/10.1063/1.4751336

Challenge us.

What are your needs for periodic signal detection?

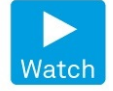

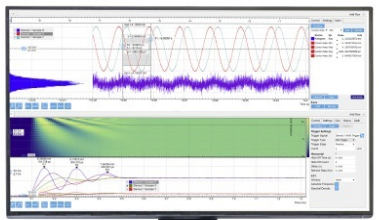

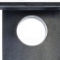

Zurich

- Instruments 


\title{
AIN photonic crystal nanocavities realized by epitaxial conformal growth on nanopatterned silicon substrate
}

\author{
D. Néel, ${ }^{1, a)}$ S. Sergent, ${ }^{2,3}$ M. Mexis, ${ }^{4}$ D. Sam-Giao, ${ }^{5}$ T. Guillet, ${ }^{4}$ C. Brimont, ${ }^{4}$ \\ T. Bretagnon, ${ }^{4}$ F. Semond, ${ }^{2}$ B. Gayral, ${ }^{5}$ S. David, ${ }^{1}$ X. Checoury, ${ }^{1}$ and P. Boucaud ${ }^{1}$ \\ ${ }^{1}$ Institut d'Électronique Fondamentale, UMR CNRS 8622, Université Paris Sud-11, F-91405 Orsay, France \\ ${ }^{2}$ CRHEA-CNRS, Rue Bernard Grégory, 06560 Valbonne, France \\ ${ }^{3}$ Université de Nice Sophia Antipolis, Parc Valrose, F-06102 Nice Cedex 2, France \\ ${ }^{4}$ Université Montpellier 2, Laboratoire Charles Coulomb, CNRS UMR 5221, F-34095 Montpellier, France \\ ${ }^{5}$ CEA-CNRS group "Nanophysique et semiconducteurs," INAC-SP2M, CEA-Grenoble, 17 rue des Martyrs, \\ 38054 Grenoble Cedex 9, France
}

(Received 1 April 2011; accepted 9 June 2011; published online 29 June 2011)

\begin{abstract}
An original method to fabricate III-nitride photonic crystal membranes without etching of III-N materials is reported. A photonic crystal pattern is first realized in a silicon substrate. GaN quantum dots embedded in a thin AlN layer are then grown by conformal epitaxy using ammonia-based molecular beam epitaxy on the top of the patterned silicon substrate and a free-standing membrane is achieved by selective etching of the silicon substrate through the holes of the photonic crystal. Room temperature microphotoluminescence measurements show a quality factor as high as 1800 at $425 \mathrm{~nm}$ on a modified L3 cavity. Possibility to achieve lasing with this system is discussed. (C) 2011 American Institute of Physics. [doi:10.1063/1.3605592]
\end{abstract}

III-N materials (GaN, AlN, InN, and related alloys) have become the dominant materials for UV to blue-green semiconductor light sources. The success in the development of these materials has allowed to market blue, green, and white light-emitting diodes but also blue lasers. It is recognized that the achievement of compact III-N lasers integrated with the silicon technology would represent a significant advance for future developments. In parallel, recent works on photonic crystal resonators have demonstrated the potential of these structures to achieve optical modes with high quality factor (Q) values within small modal volumes, allowing the observation of phenomena such as the Purcell effect, ${ }^{1}$ strong coupling, ${ }^{2}$ and low-threshold lasing. ${ }^{3}$

It is expected that the combination of both III-N materials and photonic crystal membranes could lead to advanced optoelectronic devices in the UV-visible range operating at room temperature. Nevertheless, only few works have been reported on the fabrication of (III-N)-based photonic crystal membrane emitters. Indeed, nitride-based high-quality photonic crystal membranes are not easy to fabricate because the chemical inertness of III-N materials make their processing difficult. Moreover, photonic crystal structures optically active at short wavelengths require a very short lattice constant, typically between 100 and $200 \mathrm{~nm}$ and hole sizes between 50 and $150 \mathrm{~nm}$. The first III-N photonic crystals were obtained with layers grown on sapphire substrates. ${ }^{4,5}$ III-N photonic crystal surface emitting lasers at blue-violet wavelengths were demonstrated by electrical pumping of structures grown on GaN substrates ${ }^{6}$ or under optical pumping for structures combining the slow modes of a two-dimensional photonic crystal coupled with a one-dimensional Bragg mirror. ${ }^{7}$ Convex air-bridge AlN nanocavities processed on $\mathrm{SiC}$ substrate have been fabricated by photoelectrochemical etching leading to quality factors up to 2400 at 383

${ }^{a)}$ Electronic mail: delphine.neel@u-psud.fr. nm for a long $L 7$ cavity. ${ }^{8}$ Two-dimensional photonic crystal GaN suspended membranes have been reported on silicon substrate but without any optical characterization. ${ }^{9}$ Moreover, there are no reports on AlN photonic crystal membranes containing emitters like quantum dots integrated on a silicon substrate. Integration on silicon is however of tremendous importance for the development of low cost optoelectronic devices in the blue-UV range.

In this letter, we report on the fabrication and the study of AlN photonic crystal membrane nanocavities with the embedded GaN quantum dots (QDs) for UV-visible emission at room temperature. To avoid problems encountered with the AlN etching, an original fabrication process of III-N photonic crystal membranes was developed. A nitride photonic crystal membrane is realized by performing a two-dimensional conformal nitride epitaxial growth by ammonia-based molecular beam epitaxy $\left(\mathrm{NH}_{3}-\mathrm{MBE}\right)$ on a patterned silicon substrate followed by selective etching between silicon and III-N material. This innovative technique relies on the silicon technology processing and circumvents the difficulty associated with the etching of III-N materials.

The studied photonic crystal samples were fabricated by using four main processing steps. First, a silicon substrate is nanopatterned following a triangular lattice of air holes (Fig. 1(a)). A lattice period $a=170 \mathrm{~nm}$ is chosen in order to obtain a photonic band gap around $425 \mathrm{~nm}$ for a final hole radius after processing $r=0.23 a(\sim 40 \mathrm{~nm})$. A $90 \mathrm{~nm}$-thick layer of ZEP-520A resist is spun directly on a (111) silicon substrate and is insolated with a $30 \mathrm{keV}$ Raith e-beam lithography system. Patterns are then transferred to silicon by inductively coupled plasma etching in two steps. First, the pattern is initiated by an anisotropic etching. The holes are then etched using the Bosch process that consists in switching periodically between an $\mathrm{SF}_{6}$ etching phase and $\mathrm{C}_{4} \mathrm{~F}_{8}$ sidewall passivation phase. This process is very selective and allows to use thin resist thickness, which is technologically 
(a)

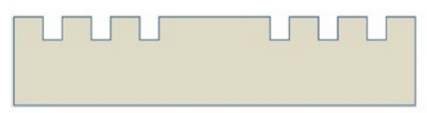

(b)

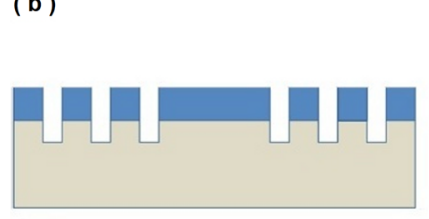

(e)

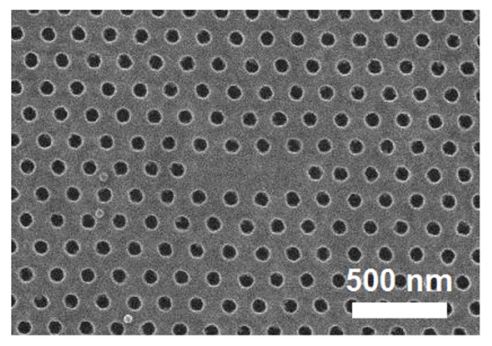

FIG. 1. (Color online) Schematic view of the fabrication steps. (a) Processing of photonic crystal in silicon (111) substrate by electronic lithography and inductively coupled plasma etching. (b) Conformal growth of $15 \mathrm{~nm}$ of AlN by $\mathrm{NH}_{3}$-MBE on the nanopatterned substrate. (c) Underetching of the silicon substrate by HNA etching. (d) $\mathrm{NH}_{3}$-MBE conformal growth of 85 $\mathrm{nm}$ of AlN with two GaN QDs planes. (e) Scanning electron microscope image of a modified $L 3$ photonic crystal membrane nanocavity. The period and the hole radii of the AlN photonic crystal are $170 \mathrm{~nm}$ and $40 \mathrm{~nm}$, respectively. The side holes of the cavity are moved to the edge by $d=0.2 a$ to increase resonance efficiency.

compatible with the small size of the desired nanopatterns. In a second step, a $15 \mathrm{~nm}$-thick AIN layer is conformally grown on the patterned silicon substrate by $\mathrm{NH}_{3}$-MBE (Fig. (b)). ${ }^{10,11}$ We emphasize that the nanopatterning does not degrade the silicon surface and good quality epitaxial growth is achieved. The silicon is then underetched by a HF/nitric acid/acetic acid (HNA) solution in order to release the strain in the structure and to fabricate a membrane (Fig. 1(c)). An $85 \mathrm{~nm}$-thick layer of AlN is then further conformally grown on the patterned substrate by $\mathrm{NH}_{3}$-MBE, the total AlN thickness corresponding to a $\lambda / 2 \mathrm{n}$ layer at $425 \mathrm{~nm}$. Two planes of GaN quantum dots separated by a $10 \mathrm{~nm}$ AlN spacer are inserted into the middle of the AlN layer during the epitaxy (Fig. 1(d)). Controlled growth of very thin GaN QD epilayers on silicon substrates and their high emission efficiency at room temperature around $400 \mathrm{~nm}$ have previously been evidenced. ${ }^{10,11}$ This fabrication process has been optimized taking into account that some parasitic material deposition occurs on the vertical silicon side walls. That could prevent a good underetching step. It explains why the epitaxial growth is interrupted after a first very short growth step (almost no side wall parasitic material deposition) and then the longer second growth step is carried out only after the underetching step. The underetching depth is around $750 \mathrm{~nm}$, a thickness sufficiently large to ensure a decoupling of the optical mode from the substrate. Various types of nanocavities were fabricated, in particular $L 3$ modified cavities. The $L 3$ modified cavities are defined by three missing air holes with the edge air holes laterally shifted by a fraction of the lattice parameter $(d=0.2 a) .{ }^{12}$ Figure $1(\mathrm{e})$ shows a scanning electron microscope image of such a membrane cavity.
Microphotoluminescence ( $\mu$-PL) measurements were performed using a non-commercial microscope with a spot diameter of approximately $1.5 \mu \mathrm{m}$ (power density $50 \mathrm{~W}$ $\left.\mathrm{cm}^{-2}\right){ }^{13}$ For quality factor measurements, the $\mu$-PL signal was dispersed by a high-resolution spectrometer (3600 lines/ $\mathrm{mm}, 55 \mathrm{~cm}$ focal length) with a spectral resolution of $\sim 0.16$ $\mathrm{meV}$. Spectroscopic measurements were performed at roomtemperature with a $266 \mathrm{~nm} \mathrm{CW}$ laser (Crylas/FQCW 266-50) and a spectrometer with a liquid nitrogen-cooled CCD. Figure 2 shows the room-temperature photoluminescence spectrum of a modified $L 3$ cavity. Three resonant modes are evidenced around $2.910 \mathrm{eV}, 2.975 \mathrm{eV}$, and $2.995 \mathrm{eV}$. Similar measurements were performed on a standard $L 3$ cavity. Without edge holes displacement, the lowest energy mode is hardly observed and the higher-energy modes are spectrally shifted, in agreement with modeling (not shown). These resonances are thus not associated with the slow-modes of the photonic crystal but with the resonant modes of the cavity. A zoom of the spectrum of the fundamental mode with the highest quality factor is shown in the inset of Fig. 2. A quality factor of 1800 is achieved at $2.912 \mathrm{eV}(425 \mathrm{~nm})$. The small shift in the peak position between the two measurements can be likely explained by thermal effects occurring during the measurements, especially at high excitation power. Also, it was observed that the quality factor decreases as the pumping power is increased because of additional absorption losses due to free carriers photocreated in the membrane. ${ }^{14}$

For a better understanding of these results, the cavity was simulated using three-dimensional finite-difference in time domain calculations (3D-FDTD). ${ }^{15,16}$ Dimensions used in the calculations were those obtained from the scanning electron microscope images. We used a refractive index of 2.15 for the AlN at $400 \mathrm{~nm} .{ }^{17}$ The 3D-FDTD spectrum is shown in Fig. 3. Three resonant modes are calculated for the modified $L 3$ cavity at energies $2.847 \mathrm{eV}, 2.932 \mathrm{eV}$, and 2.955 $\mathrm{eV}$, along with a broader emission at $3.05 \mathrm{eV}$. Their quality factors are 4810,485 , and 1370 , respectively, as compared to

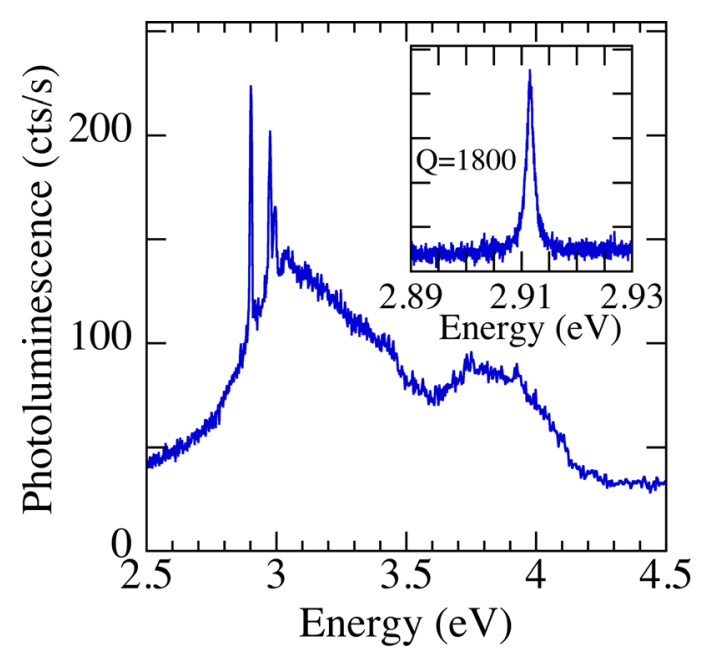

FIG. 2. (Color online) Experimental room temperature spectrum of a modified $L 3$ nanocavity with a lateral shift $d=0.2 a$. The high-energy recombination feature, around $3.8 \mathrm{eV}$, is associated with lowest-height QD emission. The low-energy recombination component, around $3 \mathrm{eV}$, corresponds to large-height QD emission. Inset: spectrum with a high resolution grating (3600 lines/mm) of the lowest-energy mode. A Q factor of 1800 is obtained. 


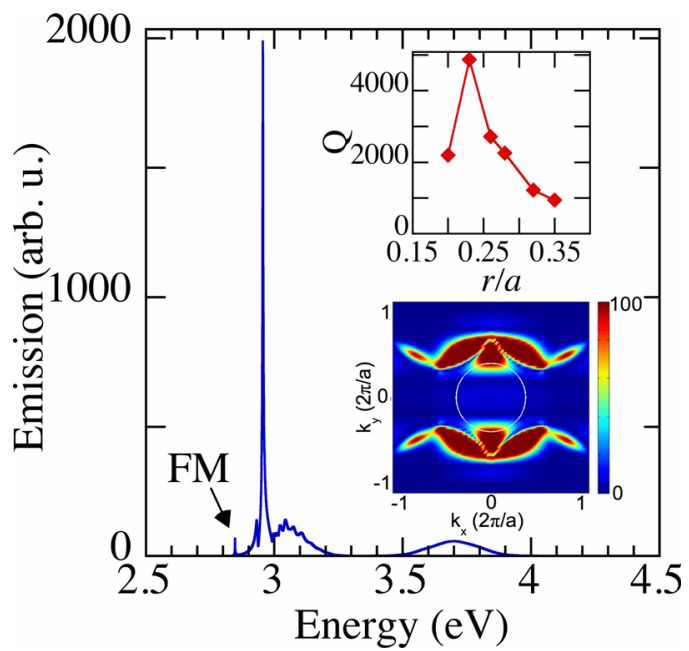

FIG. 3. (Color online) 3D-FDTD spectrum of the modified $L 3$ nanocavity. The fundamental mode (FM) is indicated by an arrow. The top inset shows the evolution of the $\mathrm{Q}$ factor of the fundamental mode as a function of the hole radius. The bottom inset shows the two-dimensional Fourier-transform of the electric field distribution of the fundamental mode. The leaky region or light cone is highlighted.

1800,270 , and 270 experimentally. The spectrum is dominated by the mode at $2.955 \mathrm{eV}$, the two other modes having in the modeling a significantly smaller amplitude. A satisfying agreement is obtained between experiments and modeling for the energy position of the modes and their energy spacing. Since Fig. 3 represents the spectrum of the simulated field on the top of the membrane, the amplitude of each component is different from the experimental one that is indeed a far field spectrum spatially filtered by the microscope objective. ${ }^{18}$ Experimentally, the quality factor of the fundamental mode is only 2.5 times smaller than the calculated one. We attribute the discrepancy to the inhomogeneities of the air holes, to the hole roughness, and to the reduced symmetry of the holes, which are not perfect circles. We emphasize that the calculated quality factors for AlN membrane cavities are significantly smaller than those that can be obtained with silicon photonic crystal membrane-type L3 cavities (typically 100 000 for optimized structures). ${ }^{14,19}$ Indeed, the smaller value of the refractive index of AIN as compared to silicon (2.15 vs. 3.45) decreases significantly the photonic band gap width. Moreover, the associated normalized frequency is around 0.4 instead of 0.25 , and the Fourier components inside the air escape cone are significantly larger as compared to silicon membranes, leading to a decreased quality factor (see inset of Fig. 3). The quality factor of the fundamental mode is very sensitive to the cavity design and to the air hole radius (second inset of Fig. 3). A sharp maximum is obtained for a radius $r=0.23 a$ corresponding to the investigated structure. Experimentally, we have measured a quality factor of 1170 at $417 \mathrm{~nm}$ for a $r / a$ value of 0.25 , corresponding to a $35 \%$ decrease from the maximum, consistent with the modeling.

One possible prospect for such small volume/high Q cavities would be to obtain a UV nanolaser. Taking into account the Purcell effect in the framework of rate equations for the cavity/QD system, the lasing conditions reads $\xi=\frac{N_{t} \beta}{2 \tau_{e} \gamma_{c}}>0.25$, where $\mathrm{N}_{\mathrm{t}}$ is the number of QDs that spatially and spectrally match the mode, $\beta$ is the spontaneous emission coupling factor to the cavity mode, $\tau_{\mathrm{e}}$ is the average spontaneous emission rate of the QDs in the mode, and $\gamma_{c}$ is the photon escape rate out of the cavity. ${ }^{20}$ For this lasing evaluation, the homogeneous linewidth is not a critical parameter: as the homogeneous linewidth increases, the decrease of the Purcell effect is compensated by the increased number of dots resonant with the optical mode. It is however now well known that, in the case of very small QD-based lasers showing a large Purcell effect, the mere rate equations do not take into account all the processes leading to gain and that lasing can actually be achieved with $\xi$ values smaller than predicted as shown in Ref. 21 for which lasing is achieved with $\xi=6 \times 10^{-2}$. In the present case, $\mathrm{N}_{\mathrm{t}}$ is around 2 , the Purcell factor is about 200, leading to $\beta \sim 1$. The natural spontaneous emission rate for such large GaN QDs undergoing a strong quantum confined Stark effect is about $200 \mathrm{~ns}$ for an emission at $3 \mathrm{eV}, 22$ yielding an average spontaneous emission rate $\tau_{\mathrm{e}}$ due to the Purcell effect of $4 \mathrm{~ns}$, taking into account an average decrease of the Purcell factor by 4 due to spectral and spatial mismatch between the dots and the mode. This gives $\xi=1 \times 10^{-4}$. One sees that the present cavity is far from reaching lasing conditions. However, the new process presented here should be transposable to much shorter wavelengths (around $300 \mathrm{~nm}$ emission) for which the GaN/AIN QD natural lifetime is much shorter, around $0.5 \mathrm{~ns}$. It is reasonable to expect to gain a factor of two on the QD density by increasing the number of QD planes and to increase further the quality factor to reach 4000. This would give a $\xi$ around 0.1 , which gives reasonable hope to reach UV (around $300 \mathrm{~nm}$ ) lasing in such a nanolaser.

This work was supported by the French national research agency (project SINPHONI ANR-08-NANO-021).

\footnotetext{
${ }^{1}$ A. Badolato et al., Science 308, 1158 (2005).

${ }^{2}$ T. Yoshie et al., Nature (London) 432, 200 (2004).

${ }^{3}$ M. Loncar et al., Appl. Phys. Lett. 81, 2680 (2002).

${ }^{4}$ T. N. Oder et al., Appl. Phys. Lett. 83, 1231 (2003).

${ }^{5}$ Y.-S. Choi et al., Appl. Phys. Lett. 87, 243101 (2005).

${ }^{6} \mathrm{H}$. Matsubara et al., Science 319, 445 (2008).

${ }^{7}$ T.-C. Lu et al., Appl. Phys. Lett. 93, 111111 (2008).

${ }^{8}$ M. Arita et al., Appl. Phys. Lett. 91, 051106 (2007).

${ }^{9}$ A. Rosenberg et al., J. Vac. Sci. Technol. B 25, 721 (2007).

${ }^{10}$ S. Sergent et al., Appl. Phys. Express 2, 051003 (2009).

${ }^{11}$ S. Sergent et al., J. Appl. Phys. 109, 053514 (2011).

${ }^{12}$ Y. Akahane et al., Nature (London) 425, 944 (2003).

${ }^{13}$ M. Mexis et al., Opt. Lett. 36, 2203 (2011).

${ }^{14} \mathrm{M}$. El Kurdi et al., Opt. Express 16, 8780 (2008).

${ }^{15}$ X. Checoury et al., Appl. Phys. Lett. 86, 151111 (2005).

${ }^{16}$ Z. Han et al., Opt. Comm. 283, 4387 (2010).

${ }^{17}$ N. Antoine-Vincent et al., J. Appl. Phys. 93, 5222 (2003).

${ }^{18}$ J. Vuckovic et al., IEEE J. Quantum Electron. 38, 850 (2002).

${ }^{19}$ Y. Akahane et al., Opt. Express 13, 1202 (2005).

${ }^{20} \mathrm{~J} .-\mathrm{M}$. Gérard in Photonic Crystals: Towards Nanoscale Photonic Devices, edited by J.-M. Lourtioz, H. Benisty, V. Berger, and J.-M. Gérard (Springer, Berlin, 2008).

${ }^{21}$ S. Strauf et al., Phys. Rev. Lett 96, 127404 (2006).

${ }^{22}$ T. Bretagnon et al., Phys. Rev. B 73, 113304 (2006).
} 